\title{
The DarkSide-50 Experiment: A Liquid Argøn Target for Dark Matter Particles
}

P. Agnes ${ }^{1}$, L. Agostino ${ }^{2}$, I. F. M. Albuquerque ${ }^{3,4}$, T. Alexander ${ }^{5,6}$, A. K. Alton ${ }^{7}$, K. Arisaka ${ }^{8}$, H. O. Back ${ }^{3,8}$, B. Baldin 6 , K. Biery ${ }^{6}$, G. Bonfini ${ }^{10}$, M. Bossa ${ }^{1,10}$, B. Bottino ${ }^{12,13}$, A. Brigatti ${ }^{14}$, J. Brodsky ${ }^{3}$, F. Budano ${ }^{15,16}$, S. Bussino ${ }^{15,16}$, M. Cadeddu ${ }^{17,18}$, L. Cadonati ${ }^{5}$, M. Cadoni ${ }^{17,18}$, F. Calaprice ${ }^{3}$, N. Canci ${ }^{18,10 a}$, A. Candela ${ }^{10}$, H. $\mathrm{Cao}^{3}$, M. Cariello ${ }^{13}$, M. Carlini ${ }^{10}$, S. Catalanotti ${ }^{20,21}$,

P. Cavalcante ${ }^{2,10}$, A. Chepurnov ${ }^{23}$, A. G. Cocco ${ }^{21}$, G. Covone ${ }^{20,21}$, L. Crippa ${ }^{24,14}$,

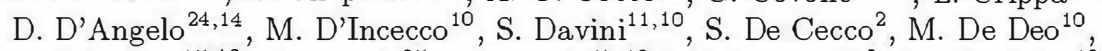
M. De Vincenzi ${ }^{15,16}$, A. Derbin ${ }^{25}$, A. Devoto ${ }^{17,18}$, F. Di Eusanio ${ }^{3}$, G. Di Pietro ${ }^{10,14}$,

E. Edkins ${ }^{26}$, A. Empl ${ }^{19}$, A. Fan ${ }^{8}$, G. Fiorillo ${ }^{20,21}$, K. Fomenko $^{27}$, G. Forster ${ }^{5,6}$,

D. Franco ${ }^{1}$, F. Gabriele ${ }^{10}$, C. Galbiati ${ }^{3,10}$, C. Giganti ${ }^{2}$, A. M. Goretti ${ }^{10}$,

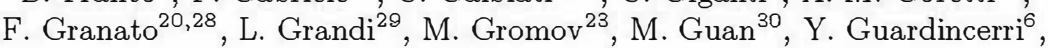

B. R. Hackett ${ }^{26}$, K. Herner ${ }^{6}$, E. V. Hungerford ${ }^{19}$, Al. Ianni ${ }^{31,10}$, An. Ianni ${ }^{3,10}$,

I. James ${ }^{15,16}$, C. Jollet ${ }^{32}$, K. Keeter ${ }^{33}$, C. L. Kendziora ${ }^{6}$, V. Kobychev ${ }^{34}$, G. Koh ${ }^{3}$,

D. Korablev ${ }^{27}$, G. Korga ${ }^{19,10}$, A. Kubankin ${ }^{35},{\text { X. } \text { Li }^{3}, \text { M. Lissia }}^{18}$, P. Lombardi ${ }^{14}$,

S. Luitz ${ }^{36}$, Y. Ma ${ }^{30}$, I. N. Machulin ${ }^{37,38}$, A. Mandarano ${ }^{11,10}$, S. M. Mari ${ }^{15,16}$,

J. Maricic $^{26}$, L. Marini ${ }^{12,13}$, C J. Martoff ${ }^{28}$, A. Meregaglia ${ }^{32}$, P. D. Meyers ${ }^{3}$, T. Miletic ${ }^{28}$, R. Milincic ${ }^{26}$, D. Montanari ${ }^{6}$, A. Monte ${ }^{5}$, M. Montuschi $^{10}$,

M. Monzani ${ }^{36}$, P. Mosteiro ${ }^{3}$, B. J. Mount ${ }^{33}$, V. N. Muratova ${ }^{25}$, P. Musico ${ }^{13}$,

J. Napolitano ${ }^{28}$, A. Nelson ${ }^{3}$, S. Odrowski ${ }^{10}$, M. Orsini ${ }^{10}$, F. Ortica ${ }^{39,40}$,

L. Pagani ${ }^{12,13}$, M. Pallavicini ${ }^{12,13}$, E. Pantic ${ }^{4}$, S. Parmeggiano ${ }^{14}$, K. Pelczar ${ }^{42}$,

N. Pelliccia ${ }^{39,40}$, S. Perasso ${ }^{1}$, A. Pocar ${ }^{5,3}$, S. Pordes ${ }^{6}$, D. A. Pugachev ${ }^{37,38}$, H. Qian ${ }^{3}$,

K. Randle ${ }^{5}$, G. Ranucci ${ }^{14}$, A. Razeto ${ }^{10,3}$, B. Reinhold ${ }^{26}$, A. L. Renshaw ${ }^{8,19}$,

A. Romani ${ }^{39,40}$, B. Rossi ${ }^{21,3}$, N. Rossi ${ }^{10}$, D. Rountree ${ }^{22}$, D. Sablone ${ }^{10}$, P. Saggese ${ }^{14}$,

R. Saldanha ${ }^{29}$, W. Sands ${ }^{3}$, S. Sangiorgio ${ }^{43}$, C. Savarese ${ }^{11,10}$, E. Segreto ${ }^{44}$,

D. A. Semenov ${ }^{25}$, E. Shields ${ }^{3}$, P. N. Singh ${ }^{19}$, M. D. Skorokhvatov ${ }^{37,38}$,

O. Smirnov ${ }^{27}$, A. Sotnikov ${ }^{27}$, C. Stanford ${ }^{3}$, Y. Suvorov ${ }^{8,10,37}$, R. Tartaglia ${ }^{10}$,

J. Tatarowicz ${ }^{28}$, G. Testera ${ }^{13}$, A. Tonazzo ${ }^{1}$, P. Trinchese ${ }^{20}$, E. V. Unzhakov ${ }^{25}$,

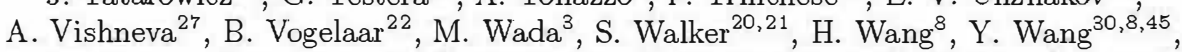
A. W. Watson ${ }^{28}$, S. Westerdale ${ }^{3}, J$. Wilhelmi ${ }^{28}$, M. M. Wojcik ${ }^{42}$, X. Xiang ${ }^{3}$, J. Xu $^{3}$, C. Yang ${ }^{30}$, J. Yoo $^{6}$, S. Zavatarelli ${ }^{13}, \mathrm{~A} \mathrm{Zec}^{5}$, W. Zhong ${ }^{30}$, C. Zhu ${ }^{3}$, and G. Zuzel ${ }^{42}$

${ }^{1} A P C$, Université Paris Diderot, CNRS/IN2P3, CEA/Irfu, Obs. de Paris, Sorbonne Paris Citée, Paris 75205, France; ${ }^{2}$ LPNHE Paris, Université Pierre et Marie Curie, Université Paris Diderot, CNRS/IN2P3, Paris 75252, France; ${ }^{3}$ Department of Physics, Princeton University, Princeton, NJ 08544, USA; ${ }^{4}$ Instituto de Fúsica, Universidade de São Paulo, São Paulo 05508-090, Brazil; ${ }^{5}$ Amherst Center for Fundamental Interactions and Dept. of Physics, University of Massachusetts, Amherst, MA 01003, USA; ${ }^{6}$ Fermi National Accelerator Laboratory, Batavia, IL 60510, USA; ${ }^{7}$ Department of Physics, Augustana University, Sioux Falls, SD 57197, USA; ${ }^{8}$ Department of Physics and Astronomy, University of California, Los Angeles, CA 90095, USA; ${ }^{9}$ Pacific Northwest National Laboratory, Richland, WA 99354, USA; ${ }^{10}$ Laboratori Nazionali del Gran Sasso, Assergi AQ 67010, Italy; ${ }^{11}$ Gran Sasso Science Institute,

This manuscript has been authored by Fermi Research Alliance, LLC under Contract No.DE-AC02-07CH11359 with the U.S. Department of Ener y, Office of Science, Office of High Enery Physics. The U.S. Government retains and the publisher, by acceptin the article for publication, acknowled es that the U.S. Government retains a non-exclusive, paid-up, irrevocable, world-wide license to publish or reproduce the published form of this manuscript, or allow others to do so, for U.S. Government purposes. 
L'A uila AQ 67100, Italy; ${ }^{12}$ Department of Physics, Università degli Studi, Genova 16146, Italy; ${ }^{13}$ Istituto Nazionale di Fisica Nucleare, Sezione di Genova, Genova 16146, Italy; ${ }^{14}$ Istituto Nazionale di Fisica Nucleare, Sezione di Milano, Milano 20133, Italy; ${ }^{15}$ Istituto Nazionale di Fisica Nucleare, Sezione di Roma Tre, Roma 00146, Italy; ${ }^{16}$ Department of Physics and Mathematics, Università degli Studi Roma Tre, Roma 00146, Italy; ${ }^{17}$ Department of Physics, Università degli Studi, Cagliari 09042, Italy; ${ }^{18}$ Istituto Nazionale di Fisica $\mathrm{Nu}$ cleare, Sezione di Cagliari, Cagliari 09042, Italy; ${ }^{19}$ Department of Physics, University of Houston, Houston, TX 77204, USA; ${ }^{20}$ Department of Physics, Università degli Studi Federico II, Napoli 80126, Italy; ${ }^{21}$ Istituto Nazionale di Fisica Nucleare, Sezione di Napoli, Napoli 80126, Italy; ${ }^{22}$ Department of Physics, Virginia Tech, Blacksburg, VA 24061, USA; ${ }^{23}$ Skobeltsyn Institute of Nuclear Physics, Lomonosov Moscow State University, Moscow 119991, Russia; ${ }^{24}$ Department of Physics, Università degli Studi, Milano 20133, Italy; ${ }^{25}$ St. Petersburg Nuclear Physics Institute NRC Kurchatov Institute, Gatchina 188350, Russia; ${ }^{26}$ Department of Physics and Astronomy, University of Hawai'i, Honolulu, HI 96822, USA; ${ }^{27}$ Joint Institute for Nuclear Research, Dubna 141980, Russia; ${ }^{28}$ Department of Physics, Temple University, Philadelphia, PA 19122, USA; ${ }^{29}$ Kavli Institute, Enrico F'ermi Institute, and Dept. of Physics, University of Chicago, Chicago, IL 60637, USA; ${ }^{30}$ Institute of High Energy Physics, Beïing 100049, China; ${ }^{31}$ Laboratorio Subterráneo de Canfranc, Canfranc Estación 22880, Spain; ${ }^{32} I P H C$, Université de Strasbourg, CNRS/IN2P3, Strasbourg 67037, France; ${ }^{33}$ School of Natural Sciences, Black Hills State University, Spearfish, SD 57799, USA ${ }^{34}$ Institute for Nuclear Research, National Academy of Sciences of Ukraine, Kiev 03680, Ukraine; ${ }^{35}$ Radiation Physics Laboratory, Belgorod National Research University, Belgorod 308007, Russia ; ${ }^{36}$ S.LAC National Accelerator Laboratory, Menlo Park, CA 94025, USA; ${ }^{37}$ National Research Centre Kurchatov Institute, Moscow 123182, Russia; ${ }^{38}$ National Research Nuclear University MEPhI, Moscow 115409, Russia; ${ }^{39}$ Department of Chemistry, Biology and Biotechnology, Università degli Studi, Perugia 06123, Italy; ${ }^{40}$ Istituto Nazionale di Fisica Nucleare, Sezione di Perugia, Perugia 06123, Italy; ${ }^{41}$ Department of Physics, University of California, Davis, CA 95616, USA; ${ }^{2}$ Smoluchowski Institute of Physics, Jagiellonian University, Krakow 30348, Poland; ${ }^{43}$ Lawrence Livermore National Laboratory, Livermore, CA 94550, USA; ${ }^{44}$ Institute of Physics Gleb Wataghin, Universidade Estadual de Campinas, São Paulo 13083-859, Brazil; ${ }^{45}$ School of Physics, University of Chinese Academy of Sciences, Beijing 100049, China. 


\begin{abstract}
The DarkSide-50 experiment, located at the "Laboratori Nazionali del Gran Sasso (INFN)", is based on low-radioactivity argon double phase time projection chamber, surrounded by an active liquid scintillator veto, designed for the zero background achievement. The liquid argon features sufficient self shielding and easy scalability to multi-tons scale. The impressive reduction of the ${ }^{39} \mathrm{Ar}$ isotope (compared to the atmospheric argon), along with the excellent pulse shape discrimination, make this technology a possible candidate for the forthcoming generation of multi-ton Dark Matter experiments.
\end{abstract}

\title{
Introduction
}

Astrophysical and Cosmological observations suggest the existence of a relevant amount of dark matter in the Universe [1]. According to some theoretical models, such a matter consists of weakly interactive massive particle (WIMP) [2] and interacts with target nuclei of experiments releasing energies of order few tens of keV. The DarkSide-50 experiment, after the promising results of its predecessor DarkSide-10 [3], searches for such nuclear recoils (NR) using a liquid argon double-phase Time Projection Chamber (LAr-TPC), surrounded by a liquid scintillator veto (LSV) located in the middle of a water Cherenkov veto (WCV), both used for rejecting the coincidences in the LAr-TPC induced by cosmic and material radiation (technical details are reported in [4-7]). Two arrays of 19 3" PMTs, facing from the top and the bottom the liquid argon active volume $(\sim 46.4 \mathrm{~kg})$, detect the primary scintillation (S1) and the gas scintillation from drifted ionization electrons (S2). Thanks to S1 and S2 it is possible to reconstruct the full $3 \mathrm{D}$ position of the events. LAr intrinsic scintillation features allow us to reject electron recoils ( $\beta$ and $\gamma$ events from background) at the level of $1.5 \times 10^{7}$ or even better [6]. The pulse shape discrimination between $\mathrm{ER}$ and $\mathrm{NR}$ is based on the $\mathrm{f}_{90}$ parameter, i.e. the fraction of $\mathrm{S} 1$ detected in the first $90 \mathrm{~ns}$.

The DarkSide-50 experiment exploited two important data taking campaigns: first, the atmosheric argon (AAr) campaign, in which the main features of the detector have been tested [6]; second, the underground depleted argon (UAr) campaign in which the predicted characteristics have been confirmed and the impressive reduction of the ${ }^{39} \mathrm{Ar}$ has been proven [5]. The main features of both LAr-TPC and LSV detectors have been understood also by means of intense calibration campaigns with internal $\left({ }^{83 m} \mathrm{Kr}\right)$ and external $(\gamma, \mathrm{AmBe})$ sources.

\section{The Underground Argon}

UAr was extracted and purified with an effort lasted many years [8]. Fig. 1 shows a comparison between AAr and UAr spectrum in terms of S1 in a reduced volume $(34 \mathrm{~kg})$. The activity of ${ }^{39} \mathrm{Ar}$ and ${ }^{85} \mathrm{Kr}$ is determined through a simultaneous Monte Carlo (MC) fit of S1 spectrum (with field on and off) and $z$-position distribution of the corresp onding events. The ${ }^{39} \mathrm{Ar}$ activity of 
$\mathrm{UAr}$ is a factor $(1.4 \pm 0.2) \times 10^{3}$ lower than the AAr one, corresponding to $(0.73 \pm 0.11) \mathrm{mBq} / \mathrm{kg}$.

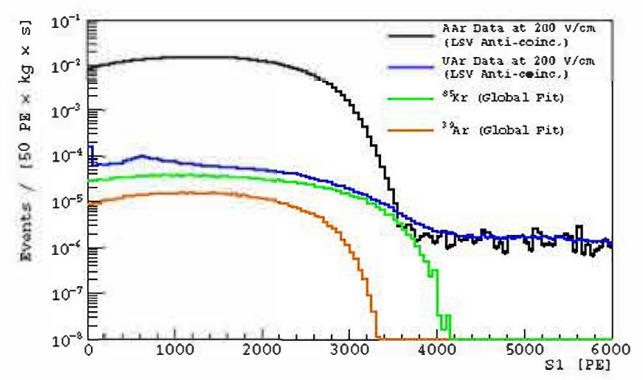

Figure 1: Livetime normalized S1 pulse integral comparison between AAr (black) and UAr (blue) events. For UAr events ${ }^{85} \mathrm{Kr}$ (green) and ${ }^{39} \mathrm{Ar}$ (orange) are shown separately [5].

The vetoing efficiency of the LSV, necessary to exclude NR events due to radiogenic or cosmogenic neutrons, during the UAr campaign is estimated to be $>99.1 \%$, using the $\mathrm{AmBe}$ calibration data and $\mathrm{MC}$ simulations.

Fig. 2a shows all UAr events in the dark matter region in the S1-fop plane after applying all selection criteria (see for details [5]). NR acceptance is based on the median of $f_{90}$ measured in SCENE experiment [9] combined with a suitable statistical model [10]. Finally, Fig. 2b shows the spin-independent WIMP-nucleon cross section 90\% CL exclusion plots for DarkSide-50 AAr and UAr (independently and combined), compare with the most popular ark matter limits [11].

\section{Future developments}

LAr-TPC based on UAr is an excellent candidate for the next generation of dark matter search experiments, thanks especially to: low background, powerful pulse shape discrimination, sufficient self-shielding and good light yield. The DarkSide collaboration is proposing, in the same underground laboratory, the DarkSide-20k with an active mass of $\sim 20 \mathrm{t}$. Yet this scaled version will be surrounded by a LSV inside a cylindrical WCV. Thanks to ultra-low background, this detector will be able to reach the sensitivity of $\sim 10^{-47} \mathrm{~cm}^{2}$ for WIMP of $1 \mathrm{TeV} / \mathrm{c}^{2}$ mass. SiPM light sensors will take the place of the usual PMTs, with a consequent increasing of the light collection. In parallel the "Urania" project, an improved extraction plant in Colorado, will guarantee an UAr production rate of $\sim 100 \mathrm{~kg} /$ day. Finally the "Aria" project, a cryogenic distillation facility for isotopic separation of argon at the Seruci mine in 


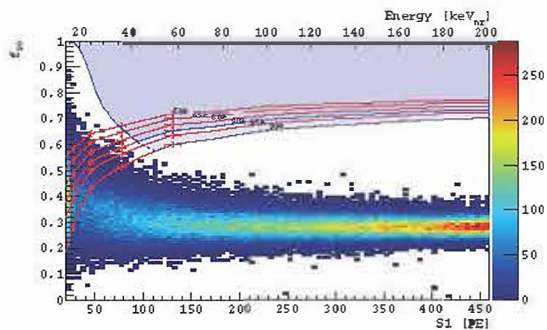

(a) Event distribution in the $\mathrm{f}_{90}-\mathrm{S} 1$ plane NR accenpance bands are derived from SCENE experiment. Shaded area represents the WIMP search region [5].

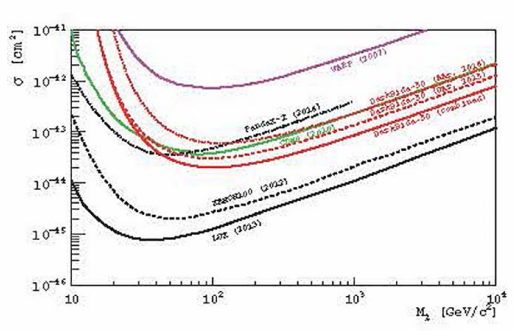

(b) Comparison between the DarkSide Spin-Independent WIMP-nucleon cross section $90 \% \mathrm{CL}$ exclusion plot and the most popular experimental limits [5].

Sardinia will further reduce the ${ }^{39} \mathrm{Ar}$ content and strongly suppress the ${ }^{85} \mathrm{Kr}$ presence.

[1] S. M. Faber and J. Gallagher, Annu. Rev. Astro. Astrophys. 17, 135 (1979); D. N. Spergel, Phys. Rev. D 37, 1353 (1988); D. Clowe et al., Ap. J. 648, L109 (2006)

[2] J. L. Feng, Annu. Rev. Astro. Astrophys. 48, 495 (2010).

[3] T. Alexander et al. [DarkSide Collaboration], Astropart. Phys. 49 (2013) 44 doi:10.1016/j.astropartphys.2013.08.004 [arXiv:1204.6218 [astro-ph.IM]].

[4] P. Agnes et al. [DarkSide Collaboration], JINST 11 (2016) no.03, P03016 doi:10.1088/1748-0221/11/03/P03016 [arXiv:1512.07896 [physics.ins-det]].

[5] P. Agnes et al. [DarkSide Collaboration], arXiv:1510.00702 [astroph.CO].

[6] P. Agnes et al. [DarkSide Collaboration], Phys. Lett. B 743 (2015) 456 doi:10.1016/j.physletb.2015.03.012 [arXiv:1410.0653 [astro-ph.CO]].

[7] P. Agnes et al. [DarkSide Collaboration], arXiv:1412.2969 [astro-ph.IM].

[8] D. Acosta-Kane et al., Nucl. Inst. Meth. A 587, 46 (2008); H. O. Back et al., arXiv:1204.6024v2 (2012); H. O. Back et al., arXiv:1204.6061v2 (2012); J. Xu et al., Astropart. Phys. 66, 53 (2015).

[9] T. Alexander et al. (The SCENE Collaboration), Phys. Rev. D 88, 092006 (2013); H. Cao et al. (The SCENE Collaboration), Phys. Rev. D 91, 092007 (2015).

[10] D. V. Hinkley, Biometrika 56, 635 (1969); M. G. Boulay and A. Hime, Astropart. Phys. 25, 179 (2006).

[11] D. S. Akerib et al. (The LUX Collaboration), arXiv:1512.03506v1 (2015); E. Aprile et al. (The XENON100 Collaboration), Phys. Rev. Lett. 109, 181301 (2012); X. Xiao et al., Phys. Rev. D 92, 052004 (2015); R. Agnese 
et al. (The SuperCDMS Collaboration), Phys. Rev. D 92, 072003 (2015); C. Amole et al. (The PICO Collaboration), Phys.Rev. Lett. 114, 231302 (2015); D. Y. Akimov et al., Phys. Lett. B 709, 14 (2012); P. Benetti et al. (The WArP Collaboration), Astropart. Phys. 28, 495 (2008). 